\title{
Keeping governments accountable: the COVID-19 Assessment Scorecard (COVID-SCORE)
}

\author{
Many actors in the response to COVID-19 are holding out for a vaccine to be developed. But in the meantime, \\ tried and tested public-health measures for controlling outbreaks can be implemented. A scorecard can be used to \\ assess governments' responses to the outbreak. \\ Jeffrey V. Lazarus, Agnes Binagwaho, Ayman A. E. El-Mohandes, Jonathan E. Fielding, Heidi J. Larson, \\ Antoni Plasència, Vytenis Andriukaitis and Scott C. Ratzan
}

$\mathrm{M}$ any government leaders and citizens are pinning their hopes on the rapid development and deployment of a vaccine against COVID-19. So far, considerable progress has been made toward identifying effective vaccines against COVID-19, and more than 100 candidates are now being evaluated ${ }^{1}$.

However, successful vaccines typically take years to develop. A vaccine against human immunodeficiency virus has eluded medical science for more than 30 years now, and past attempts to create vaccines against other coronaviruses, such as the agent that causes severe acute respiratory syndrome (SARS), have highlighted the challenging and time-consuming nature of vaccine research and development ${ }^{2-5}$. Moreover, the virus that causes COVID19, SARS-CoV-2, may well mutate; the eventual vaccine may provide only partial protection; and, notably, vaccine hesitancy may preclude large-scale uptake and the development of herd immunity ${ }^{6}$. Realistically, the world cannot count on a vaccine breakthrough to decisively end the COVID-19 pandemic any time soon.

\section{Tried and tested tools}

Much about the COVID-19 crisis is not new. Pandemics have ravaged the world since time immemorial, and COVID-19 will hardly be the last. Medicine and public health have had a string of remarkable successes in controlling deadly diseases such as smallpox, polio and measles. However, these successes took many years and required large-scale global mobilization involving the World Health Organization (WHO) and international coordination mechanisms.

Several effective measures to strengthen national preparedness for health emergencies have been identified through trial and error, and since the late $1940 \mathrm{~s}^{7}$, countries have committed to undertake such measures, including being signatories of the International Health Regulations ${ }^{8,9}$.

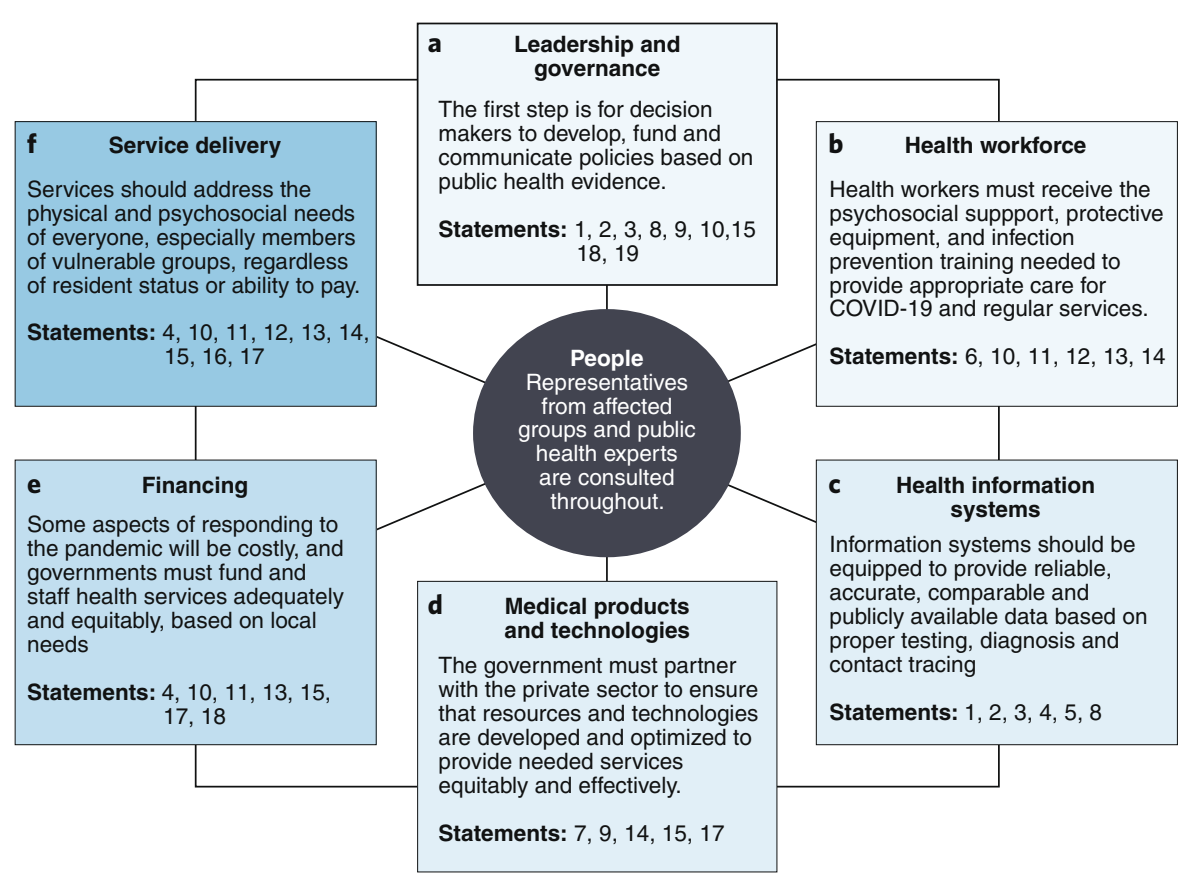

Fig. 1 | A pandemic health systems framework. Adapted from the Nobody Left Outside (NLO) Service Design Checklist ${ }^{28}$, which is based on the WHO Health Systems Framework (2007). Statement numbers correspond to the COVID-19 Assessment Scorecard (Fig. 2).

The response to COVID-19 has underscored the need for governments to improve their outbreak preparedness and response by incorporating a health-systems approach. We have accordingly drawn up the following recommendations for government action based on the WHO's health systems framework (Fig. 1) and other national and international public-health guidance.

\section{Improve public health communication} and health literacy. An outbreak cannot be controlled without broad public support. Clear and consistent communication to the public via reliable, comprehensive and up-to-date data is crucial for effective outbreak control. Evidence-based communication can help foster transparency and trust, enabling the population and government officials to make informed decisions. Explaining the basis for the latest public-health recommendations particularly in the context of a novel virus, for which new evidence continues to emerge and guidelines continue to evolve - will be crucial for building trust and encouraging compliance with the recommended measures.

When a communication strategy is drawn up, varying educational, cultural and language backgrounds must be taken into account so all population groups are engaged. Consultation with public-health experts, opinion leaders and community representatives at all levels is critical for 


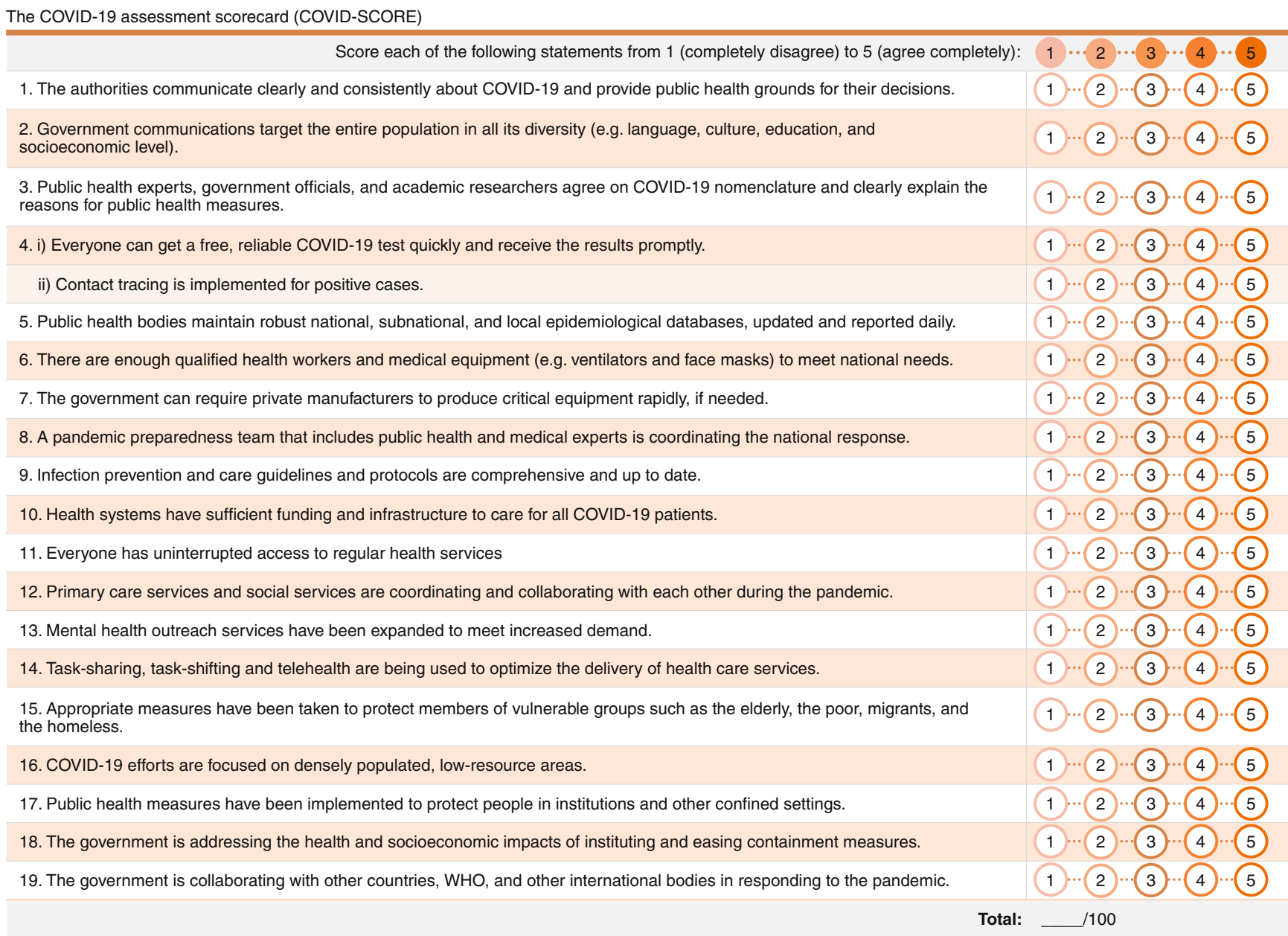

Note: For comparing national scores, a numerical multiplier should be considered (e.g. the four World Bank income classifications). For use by the general public, a short form of this scorecard (COVID-SCORE-10) may be more appropriate, using the following statements: 1, 2, 4i, 4ii, 5, 7, 11, 15, 18 , and 19.

Fig. 2 | The COVID-19 Assessment Scorecard (COVID-SCORE).

the development of appropriate strategies and will help earn the support of local stakeholders. A strategy for increasing vaccine literacy will be crucial for improving uptake, should a vaccine become available. For improvement of strategy effectiveness, it is also important to regularly survey public knowledge, perceptions and behaviors and to conduct qualitative research at a national and, if practicable, international level ${ }^{10}$.

\section{Facilitate robust surveillance and reporting. Previous outbreaks have} demonstrated the need for extensive and accurate testing to identify infected people and hot spots, as well as to better understand transmission routes and dynamics.

Small-scale random testing can provide a more accurate picture of disease prevalence ${ }^{11}$, while a rigorous testing strategy is key to guiding elimination strategies, as has already been seen with COVID-19 ${ }^{12}$. Testing should be followed by contact tracing ${ }^{13}$, although this needs to be voluntary and protect individual rights to privacy ${ }^{14}$.

Public-health bodies need to maintain real-time national, subnational and local epidemiological databases with harmonized data on every known case of infection. This will make it easier to identify clinical and behavioral factors associated with the disease and, in turn, the people and communities most at risk. Moreover, these databases will help national and international public-health planners to design interventions targeting specific epidemic profiles ${ }^{11}$ and set up proper national and international reporting if they are not already in place.

\section{Develop pandemic preparedness. Every} country should maintain two national resource pools for pandemic response: a human resource pool of appropriately qualified health workers or other people with quickly transferable skills, and a physical resource pool with items such as ventilators, masks, testing swabs and reagents. These pools need to be developed and maintained in accordance with protocols for nationally and internationally coordinated deployment to the places with the most-urgent resource needs, nationally and internationally, before future disease outbreaks become pandemics. Competition for outbreak-control resources within and among countries is unethical, and measures should be instituted to ensure equitable distribution.

Protocols should be developed for enjoining private manufacturers to rapidly produce critical equipment such as ventilators and personal protective equipment in the event of a pandemic. National and international supply chains should be strengthened, and essential items should be stockpiled to ensure an adequate supply and allow the equitable distribution of such materials to the areas of greatest need during a pandemic. Specifications, 
standards and quality-control measures for these products should be established in alignment with global standards.

Every country and international health organization should have a pandemic preparedness team of public-health and medical experts and provide pandemic-response training. These teams should be charged with preparing for future pandemics and, when a potential pandemic emerges, with coordinating a swift, dependable response. Given the global dynamics of pandemics, national pandemic preparedness also means taking measures to strengthen the capacity of the WHO and other United Nations bodies and supranational entities as appropriate.

Infection-prevention guidelines and protocols in the healthcare system must be continually reevaluated and updated. All healthcare workers should receive regular training in infection prevention and control. In the case of an outbreak, the prevention protocols should require frequent testing of healthcare workers and patients for the relevant pathogen to limit exposure and minimize nosocomial transmission - a critical intervention that has yet to be implemented in most countries for COVID- $19^{15}$.

\section{Strengthen health systems.}

Health-systems strengthening has been a leitmotif in WHO strategies and policies for over a dozen years now ${ }^{16}$, but it has received scant attention from its member states. Health systems require increased funding across the board, especially for primary care, community care and mental-health services, to improve their resilience and ability to respond to a major epidemic while providing all other health services - a need that the current pandemic is driving home. Coordination and collaboration between primary-care services and social services should also be facilitated to ensure that social-service clients receive comprehensive support during health emergencies, especially the clients who are most vulnerable and those who have complex needs. The services need to be able to address spikes in domestic violence, anxiety, depression, post-traumatic stress disorder and other stress-related conditions associated with a pandemic, including stress caused by confinement or economic insecurity. Health and social workers can also be used to promote vaccine literacy to help prepare clients for an eventual vaccine and provide actual vaccinations if one becomes available.

In times of crisis, countries should consider adopting novel approaches to health-service delivery, engaging sectors outside of health. Such approaches include task-sharing and task-shifting within the scope of current roles and expanding the scope of roles to optimize care. Another important approach to consider is the broad use of telehealth technologies, which would probably necessitate additional investment in mobile services and digital health platforms, with adequate privacy provisions, as well as collaboration with the technology sector. In some settings it may be necessary to 'upskill' community health workers, including pharmacists.

5. Ensure health and social equity. During health crises, it is more critical than ever to protect members of vulnerable groups by ensuring that they are covered by social-protection programs and have access to free health and social care, which is also in line with the United Nations framework for responding to COVID-19 $9^{17,18}$.

Outbreaks are more likely to impact people with chronic illnesses, people with low income, the elderly, migrants and minority populations, the homeless, prisoners, people with disabilities and people who use drugs $^{19}$. Plans should be in place to control epidemic spread in densely populated, low-resource areas and in all types of healthcare settings, including those located in nursing homes, psychiatric facilities, drug-rehabilitation facilities, prisons, refugee camps, educational facilities and military institutions. Gender and other dimensions of vulnerability should be considered in all pandemic control measures ${ }^{20}$, as should food security ${ }^{21}$ and other issues raised by nongovernmental organizations and by the public in surveys.

\section{Ensure that confinement and de-confinement strategies are} comprehensive. In instituting or easing restrictions on movement, it is necessary to address any resulting health and socioeconomic effects. It is important to allocate resources to provide housing and financial security and to expand public spaces and public transportation to accommodate physical distancing. Special guidelines will need to be in place to safeguard the health of workers at heightened risk of infection.

Strategic diplomacy with other countries and international bodies should be initiated quickly. International cooperation and consultation - not only on cross-border issues such as travel restrictions and the procurement of medical supplies and basic goods but also on the exchange of knowledge and experience - will improve national responses and ease the transition into a post-pandemic world. The same is true when it comes to supporting the work of international bodies and using their expertise.

\section{The realities of responding to a pan- demic in $\mathbf{2 0 2 0}$}

The collective failure of governments to control the global spread of SARS-CoV-2 is disappointing but predictable. It has revealed the hollowness of countries' commitments to international collaboration on health emergencies and global health security, while the disarray apparent in many national responses points to the need for comprehensive health-sector reform. In the face of the burgeoning threat of this epidemic, the nations of the world needed to join forces and fight it together. They failed to do so.

The complacency of governments and international organizations about the inevitable emergence of virulent new pathogens, combined with more than a decade of widespread funding cuts in the name of austerity - including cuts to not only pandemic preparedness but also healthcare, public health and social services - left governments flatfooted when COVID-19 appeared. The world watched, stunned, as members of the public were unable to buy hand sanitizer, and intensive care wards overflowed with the critically ill and dying. Healthcare workers were forced to fabricate makeshift masks and to endure dangerous exposure levels, interminable shifts and traumatizing triage decisions. Billions of dollars have been allocated to vaccine research and business bailouts, while basic public-health responses, including low-cost interventions, often remain critically underfunded.

Meanwhile, even though the WHO's express purpose is "to act as the directing and co-ordinating authority on international health work" and its constitution states that it should have a major role in eradicating epidemic disease ${ }^{22}$, countries have hesitated to draw on the agency's expertise or support its leadership. Scientific and technical experts have been exchanging information and collaborating on COVID-19 research across borders, but their cooperation has been anything but systematic. Faced with frightening prospects, most countries reluctantly adopted public-health responses ${ }^{23}$ that centered on severe restrictions on movement and assembly as well as hand washing, physical distancing and contact tracing, while large-scale international collaboration never materialized. Despite the International Health Regulations ${ }^{24}$ and WHO recommendations to the contrary ${ }^{25}$, free-movement agreements, like that covering the 26 European states in the Schengen Area, were almost immediately 
suspended as borders around the world closed $^{26}$. Each country went it alone, sometimes setting up their own agencies and task forces and instituting measures that have varied tremendously in their strictness and effectiveness, while competition among and even within countries to buy personal protective equipment, tests and ventilators led to debilitating shortages in many of the places hit hardest.

It was not until 11 March 2020 that the WHO finally declared the COVID-19 outbreak a pandemic ${ }^{27}$. At that point, Iran, Italy and Spain were already overwhelmed by COVID-19, just as parts of China had been weeks earlier. Countries that had traditionally taken a leadership role in times of international crisis refused to step up, blaming other countries and even the WHO for the spread of the disease while repeatedly denying the gravity of the problem within their own borders.

\section{Accountability and global leadership} For COVID-19, countries have naturally focused on two areas of response: immediate containment and mitigation, and facilitating the speedy development of a vaccine or cure. While we absolutely agree that vaccines and treatments are critical, we believe that governments also need to prioritize the six areas of public health action outlined above to improve their response to COVID-19 and other disease outbreaks.

For these actions to be successful, it is imperative that trust be restored in the systems that support public health. That begins with a credible chain of accountability. To help achieve this goal, we have developed the COVID-19 Assessment Scorecard (COVID-SCORE), a list of 19 statements to enable anyone to conduct an easy assessment of their city, state/regional or national government's response to COVID-19 and its preparedness for other public health emergencies (Fig. 2). If used on a large scale, it could be a powerful tool for guiding government actions.

The response to COVID-19 has the potential to generate new models of preparedness and response for future public-health emergencies, including pandemics that are even more virulent. This pandemic should be a wake-up call for countries to address the most critical shortcomings in their pandemic readiness and health-system resilience in order to prevent and mitigate the effects of future catastrophes.

Jeffrey V. Lazarus (D1⿴囗十 , Agnes Binagwaho², Ayman A. E. El-Mohandes ${ }^{3}$, Jonathan E. Fielding ${ }^{4}$, Heidi J. Larson ${ }^{5}$, Antoni Plasència', Vytenis Andriukaitis ${ }^{6}$ and Scott C. Ratzan ${ }^{3}$

${ }^{1}$ Barcelona Institute for Global Health (ISGlobal), Hospital Clinic, University of Barcelona, Barcelona, Spain. ${ }^{2}$ University of Global Health Equity, Kigali, Rwanda. ${ }^{3}$ City University of New York Graduate School of Public Health \& Health Policy, New York, NY, USA. ${ }^{4}$ UCLA Fielding School of Public Health and Geffen School of Medicine, Los Angeles, CA, USA. ${ }^{5}$ London School of Hygiene and Tropical Medicine, London, UK. ${ }^{6}$, Vilnius, Lithuania.

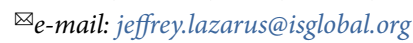

Published online: 11 June 2020

https://doi.org/10.1038/s41591-020-0950-0

\section{References}

1. World Health Organization. https://www.who.int/who documents-detail/draft-landscape-of-covid-19-candidatevaccines (2020).

2. Yamey, G. et al. Lancet 395, 1405-1406 (2020).

3. Prompetchara, E., Ketloy, C. \& Palaga, T. Asian Pac. J. Allergy Immunol. 38, 1-9 (2020).

4. Calina, D. et al. Int. J. Mol. Med. 46, 3-16 (2020).

5. Albrecht, R. et al. https://www.who.int/news-room/detail/13-042020-public-statement-for-collaboration-on-covid-19-vaccinedevelopment (accessed 19 May 2020).

6. Harrison, E. A. \& Wu, J. W. Eur. J. Epidemiol. 35, 325-330 (2020).

7. World Health Organization. Brief history of the development of the Framework on Sharing influenza viruses and access to vaccines and other benefits (World Health Organization, 2011).
8. World Health Organization. https://www.who.int/ihr/ capacity-strengthening/en/ (accessed 13 May 2020).

9. World Health Organization. https://www.who.int/ihr/about/en/ (accessed 13 May 2020)

10. Ratzan, S. C., Gostin, L. O., Meshkati, N., Parker, R. M. \& Rabin, K. NAM Perspect. https://doi.org/10.31478/202003a (2020).

11. Polonsky, J. A. et al. Philos. Trans. R. Soc. B Biol. Sci. https://doi. org/10.1098/rstb.2018.0276 (2019).

12. Organisation for Economic Co-operation and Development. http://www.oecd.org/coronavirus/ policy-responses/testing-for-covid-19-a-way-to-lift-conf inement-restrictions-89756248/ (2020).

13. Kwok, K. O. et al. Comput. Struct. Biotechnol. J. 17, 186-194 (2019).

14. Magnusson, R. Advancing the Right to Health: the Vital Role of Law Ch. 10 (World Health Organization, 2016).

15. World Health Organization. https://www.who.int/publications detail/protocol-for-assessment-of-potential-risk-factors-for2019-novel-coronavirus-(2019-ncov)-infection-among-healthcare-workers-in-a-health-care-setting (2020).

16. World Health Organization. Everybody's Business: Strengthening Health Systems to Improve Health Outcomes: WHO's Framework For Action (World Health Organization, 2007).

17. United Nations. https://unsdg.un.org/resources/un-frameworkimmediate-socio-economic-response-covid-19 (2020).

18. Binagwaho, A. Int. J. Health Policy Manag. https://www.ijhpm. com/article_3806.html (2020).

19. Jamison, D. T. et al. (eds.) Disease Control Priorities: Improving Health and Reducing Poverty. Disease Control Priorities 3rd edn., Vol. 9 (World Bank, 2018).

20. Lazarus, J. V., White, T. M., Hajo, S. \& Guy, D. https://www. isglobal.org/en_GB/-/-como-podemos-garantizar-un-enfoquesocialmente-equitativo-en-el-desconfinamiento- (2020).

21. Torero, M. Nature 580, 588-589 (2020).

22. International Health Conference. Constitution of the World Health Organization. (World Health Organization, 1946).

23. Nussbaumer-Streit, B. et al. Cochrane Database Syst. Rev. 4, CD013574 (2020).

24. World Health Organization. https://www.who.int/ihr/access/en/ (accessed 19 May 2020).

25. World Health Organization. https://www.who.int/news-room/ articles-detail/updated-who-recommendations-for-international traffic-in-relation-to-covid-19-outbreak (2020).

26. European Commission. Communication from the Commission to the European Parliament, the European Council and the Council on the second assessment of the application of the temporary restriction on non-essential travel to the EU (European Commission, 2020).

27. World Health Organization. Coronavirus disease 2019 (COVID-19) Situation Report 51 (World Health Organization, 2020).

28. Lazarus, J. V. et al. BMJ Open 10, e035621 (2020).

\section{Acknowledgements}

V. A. is a former European Commissioner for Health and Food Safety and former Minister of Health of the Republic of Lithuania.

Competing interests

The authors declare no competing interests. 